\title{
PEDAGOGIA DO ESPORTE E OBESIDADE: PERSPECTIVAS PARA UM ESTILO DE VIDA SAUDÁVEL COM BASE NA ADEQUADA INICIAÇÃO ESPORTIVA NA INFÂNCIA
}

\author{
Larissa Rafaela Galatti \\ Universidade de Campinas, Campinas, São Paulo, Brasil \\ Roberto Paes \\ Universidade de Campinas, Campinas, São Paulo, Brasil \\ Antonio Montero Seoane \\ Universidade de Campinas, Campinas, São Paulo, Brasil
}

\begin{abstract}
Resumo
Este estudo, de revisão bibliográfica, alerta para o tratamento inadequado do esporte - um dos maiores fenômenos socioculturais da contemporaneidade - na infância, o que pode ser fator de distanciamento entre o indivíduo e um estilo de vida ativo. O estudo também sinaliza para a articulação de adequados programas de esporte, em especial de Jogos Esportivos Coletivos, desde a infância, como fator que pode contribuir para um estilo de vida mais saudável e ativo, podendo auxiliar no controle do problema da obesidade ao longo da vida. Obesidade e sobrepeso são tratados, neste início de século, como um fenômeno de alcance mundial, atingindo índices de epidemia.
\end{abstract}

Palavras-chave: Pedagogia do Esporte. Saúde Pública. Obesidade. Iniciação Esportiva. Jogos Esportivos Coletivos.

\section{Introdução}

obesidade e o sobrepeso, segundo a Organização Mundial da
Saúde (OMS), são caracterizados pelo acúmulo excessivo de gordura abdominal, sendo a obesidade acompanhada de fatores de risco para a saúde. Atualmente, a obesidade é um problema mundial de saúde, cuja prevalência vem aumentando também nos países em desenvolvimento (KAIN et al., 2003; DURAZO-ARVIZU et al., 2008) e na população jovem (BUNDRED et al., 2001; STAMATAKIS et al., 2005; ADAMS et al., 2008). Entre os jovens, o que preocupa em especial é a obesidade infantil: entre 1980 e 2000, a prevalência de sobrepeso e obesidade em crianças aumentou até cinco vezes nos países desenvolvidos e até quatro vezes naqueles em desenvolvimento (WANG; MONTEIRO; POPKIN, 2002; OGDEN et al., 2006). No Brasil, a proporção de crianças e adolescentes com excesso de peso 
também cresceu de 4,1\% (1974-1975) para 13,9\% (1996-1997) (WANG; MONTEIRO; POPKIN, 2002).

No século XXI, o problema ganhou proporção epidêmica: pesquisas da OMS realizadas em 2007 estimaram que cerca de 22 milhões de crianças com até doze anos, em todo o mundo, apresentavam sobrepeso, das quais mais de $75 \%$ viviam em países com pequena ou média economia. $\mathrm{O}$ fato de a maior parte das crianças com obesidade e sobrepeso viverem em países em desenvolvimento é alarmante pela tendência a menores recursos financeiros e técnicos para o controle de doenças relacionadas ao problema, aumentando ainda mais sua incidência. Como se trata de uma questão de saúde pública, é importante a pesquisa de alternativas para mudanças nesse quadro. Nesse sentido, este artigo apresenta o exercício físico como um auxiliar relevante no controle do sobrepeso na infância e aborda, em específico, o esporte e os Jogos Esportivos Coletivos (JECs) como uma possibilidade que, se bem orientada desde a infância, pode ser um fator auxiliar no controle do sobrepeso e promoção da saúde por toda a vida.

\section{Obesidade e infância}

A obesidade está relacionada ao acúmulo excessivo de gordura corporal acompanhado de ao menos uma doença correlata, tal qual hipertensão, diabetes tipo 2 ou dislipidemias, entre outras. Já a expressão "gordura excessiva" se refere a uma condição idêntica à da obesidade, porém sem que o indivíduo apresente qualquer uma de suas consequências (MCARDLE et al., 2001).

A mensuração da obesidade pode se dar por diferentes métodos, como pesagem hidrostática, dobras cutâneas, circunferências etc. Dados da OMS indicam que cerca de $15 \%$ da população mundial é obesa, o que caracteriza epidemia mundial. Na Europa, no final do século XX (década de 1980), verificou-se em dez anos um aumento entre $10 \%$ e $40 \%$ de obesidade na maioria dos países, destacando-se a Inglaterra. Nas Américas, estudos demonstram que o padrão de obesidade para ambos os sexos vem aumentando, tanto em países desenvolvidos quanto em países em desenvolvimento (WHO, 2011).

Segundo dados da Associação Brasileira para o Estudo da Obesidade e da Síndrome Metabólica (Abeso), em 1997 mais do que 35\% da população brasileira apresentava sobrepeso ou obesidade, e estimativas atuais demonstram que o Brasil será o quinto país com o maior 
número de obesos no mundo em 2025 (WHO, 2011). De acordo com a Pesquisa de Orçamentos Familiares (POF), divulgada pelo Instituto Brasileiro de Geografia e Estatística (IBGE, 2003), os brasileiros não estão se alimentando corretamente: são 38,8 milhões de pessoas com vinte anos ou mais de idade que estão acima do peso, o que significa $40,6 \%$ da população total do país. Além do mais, dentro desse grupo, 10,5 milhões são obesos.

$\mathrm{Na}$ infância os dados também são alarmantes. Nos Estados Unidos, a obesidade é o distúrbio nutricional mais comum entre crianças e jovens, sendo também a principal causa de morbmortalidade no final do século XX (GORTMAKER et al.,1999). Dados recentes apontam que $31,5 \%$ das crianças americanas apresentam sobrepeso ou obesidade (HEDLEY et al., 2004). Um levantamento epidemiológico anual revelou que a prevalência de obesidade em crianças em idade escolar de seis a onze anos tem aumentado a uma taxa de $0,3 \%$ ao ano e praticamente dobrou na última década (NAGAI et al., 2003).

No Brasil, nas últimas décadas, observou-se um processo de transição nutricional, constatando-se que entre os anos de 1974-1975 e 1989 houve uma redução da prevalência da desnutrição infantil (de 19,8\% para 7,6\%) e um aumento na prevalência de obesidade em adultos (de 5,7\% para 9,6\%) (MONTEIRO et al., 1995). Entre adolescentes, o quadro é também alarmante: a prevalência de excesso de peso foi de $16,7 \%$ em 2002-2003, sendo o problema um pouco mais frequente em meninos $(17,9 \%)$ do que em meninas (15,4\%) (IBGE, 2003). Em ambos os sexos, a frequência do excesso de peso foi máxima em adolescentes entre dez e onze anos (cerca de 22\%), diminuindo para $12 \%-15 \%$ no final da adolescência. Entre as crianças, a relação entre meninos e meninas era invertida, mas os números também preocupam: nos anos 2002-2003 havia um obeso para cada dez meninos com excesso de peso e uma obesa para cada cinco meninas com excesso de peso (IBGE, 2003).

Os prejuízos para a saúde decorrentes da obesidade infantil não são totalmente conhecidos, mas sabe-se que o aumento da adiposidade na infância aumenta substancialmente o risco de obesidade, diabetes tipo 2, hipertensão, aterosclerose, dislipidemias, apneia obstrutiva do sono e depressão na idade adulta (BAO et al., 1995; DIETZ et al., 2004; DANIELS, 2009; LEE, 2009). Essas doenças, antes mais evidentes em adultos, já são frequentemente observadas na faixa etária mais jovem (OLIVEIRA; FISBERG, 2003). 
É agravante o fato do ganho de peso na criança tender a ser acompanhado por aumento de estatura, com consequente aceleração da idade óssea e antecipação da puberdade, o que acarreta altura final diminuída, devido ao fechamento precoce das cartilagens de crescimento. Com isso, há uma tendência para queda no aumento da estatura com manutenção do incremento de peso, favorecendo que o sobrepeso e a obesidade se mantenham e até se agravem na idade adulta (HAMMER, 1992).

Segundo Cintra (1999), a obesidade tem sido considerada um dos problemas de saúde de maior prevalência na área pediátrica. Seu combate é dificultado pela origem multifatorial: está diretamente relacionada à ingestão energética maior do que o gasto energético, em geral desde a infância; tem relação com fatores genéticos, como demonstrou o estudo de Whitaker et al. (1997) - que concluiu que quando os pais da criança são obesos a probabilidade de a criança vir a ser obesa varia de 75 a $90 \%$, enquanto quando os pais são magros a probabilidade varia de 8 a 24\%; pode estar associada também a mudanças ambientais e culturais relacionadas à inatividade física na vida diária (GORAN, 2001; MCARDLE et al., 2001; Velásquez-Meléndez et al., 2004).

A origem multifatorial do problema gera também dificuldades para o estabelecimento do seu prognóstico em longo prazo. Cintra (1999) afirma que isso tem sido uma das limitações para a adoção de programas efetivos ao combate da obesidade infantil, visto que o tratamento tradicional estruturado por meio de dieta hipocalórica tem promovido a redução do gasto energético basal, diminuindo o gasto energético geral em longo prazo. Além disso, como a criança está em fase de crescimento, a redução do peso relativo poderá ser obtida pelo aumento da sua estatura e pela manutenção do seu peso corporal, não sendo necessária, em muitos casos, a diminuição do peso absoluto.

Visto isso, a prática do exercício físico se mostra uma aliada importante no controle do sobrepeso e no combate à obesidade infantil, reduzindo problemas que se manifestam na infância e se agravam na vida adulta, tais quais doenças cardiovasculares, diabetes, lesões musculoesqueléticas e alguns tipos de câncer (WHO, 2011). A atividade física contribui no combate à obesidade infantil ao aumentar o gasto energético, diminuindo a diferença entre a ingestão e o gasto energético, além de induzir modificações metabólicas que facilitam o metabo- 
lismo de lipídios e de aumentar a massa livre de gordura no organismo, aumentando, consequentemente, o metabolismo basal (MCARDLE et al., 2001).

Evidenciado o problema da obesidade e destacado o exercício físico como um auxiliar no seu combate, trataremos das contribuições da prática esportiva e, em especial, dos JECs no controle da obesidade e do sobrepeso infantil.

\section{Aspectos pedagógicos do esporte na infância como auxiliar do controle da obesidade infantil}

Neste estudo sinalizamos que dois fenômenos característicos deste século, o esporte e a obesidade, podem estar relacionados, sendo destacado o momento da infância.

O esporte é assistido, consumido e praticado por bilhões de pessoas em todo o mundo (PAES, 2002; GALATTI, 2010), está presente em diferentes mídias a partir de sua manifestação profissional e sua prática atrai cada vez mais crianças e jovens. Para Sanmartín (2004), é um fator que afeta em grande medida o conceito de qualidade de vida, sendo destacada sua prática no momento de lazer como um catalisador da saúde física e psicológica do indivíduo, além de ser cada vez mais um favorecedor de intercâmbio social.

Entretanto, o paradigma do desempenho obrigatório e o foco no resultado competitivo vêm excluindo do processo de participação esportiva crianças e adolescentes obesos e com sobrepeso (COAKLEY, 1998; GALATTI; PAES, 2008; SILVA et al., 2009). Considerando que o sedentarismo é um fator importante no desenvolvimento de sobrepeso, obesidade e outras doenças relacionadas, defendemos como positiva a iniciação esportiva nos primeiros anos de idade escolar, desde que considerados os benefícios plurais que o esporte pode proporcionar para seus praticantes, nas dimensões biopsicossociais. Em contraponto, é necessário que se evite a especialização esportiva precoce, que tende a reduzir os objetivos da prática esportiva à conquista de resultados, podendo sobrecarregar física e psicologicamente crianças e adolescentes que se destaquem na prática do esporte, e a excluir aquelas que se mostrem menos aptas - como as obesas -, reduzindo para todos os benefícios sociais que a prática esportiva pode trazer.

A especialização esportiva precoce é um assunto muito discutido pelos estudos que sinalizam para novas tendências em pedagogia do 
esporte. Esse tema merece permanente atenção, pois ainda hoje muitas pessoas corroboram a ideia de que quanto mais cedo a criança iniciar, especializar-se e se destacar em alguma modalidade esportiva maiores serão as chances de sucesso como futura atleta da modalidade escolhida. Certamente a iniciação esportiva organizada, sistematizada e aplicada tendo em conta as características e necessidades próprias da infância é um processo positivo; no entanto, a especialização e a exigência de performance e de resultados de crianças esportistas tende a ser um processo que desestimula a própria prática esportiva, além de poder trazer problemas de saúde, inclusive psicológicos.

Para Paes (1997), esse equívoco pedagógico tende a reduzir as possibilidades educacionais do esporte na infância e tem influência no abandono precoce da prática esportiva. Isso pode acarretar na escolha por outras opções de lazer, como televisão e videogame, resultando em um estilo de vida pouco ativo, muitas vezes até a idade adulta (LEONARD et al., 2000).

A especialização esportiva precoce consiste na prematura exigência feita às crianças de que realizem os gestos técnicos da modalidade em questão com perfeição e eficiência. Esse processo antecipa fases da aprendizagem esportiva, considerando mais importante que a criança execute as tarefas do que ela seja levada a compreender o esporte e a ter prazer em sua prática. Em síntese, não respeita uma sequência didática que permita ao aluno usufruir do esporte em sua pluralidade (SILVA et al., 2009). Devido ao equívoco da especialização precoce, a iniciação esportiva, que poderia trazer inúmeros benefícios, ser prazerosa, diversificada, rica em aprendizagem motora, técnica, tática e em valores e modos de comportamento, corre o risco de tornar-se pouco atrativa e até mesmo traumática.

Ao contrário, quando bem apresentado, o esporte tende a ser prazeroso, fazendo que a criança mantenha-se na prática por tempo prolongado, acarretando benefícios de cunho biológico - como aumento no gasto calórico e controle do metabolismo basal - e socioafetivo - em especial nos JECs, sendo membro de uma equipe e se relacionando positivamente com o grupo -, e contribuindo para o combate da obesidade infantil que, como vimos, é um dos grandes desafios deste século. Quando adequados à criança e ao adolescente, os processos de iniciação e de especialização esportiva aumentam as possibilidades de a prática se manter por toda a vida, contribuindo também para o controle da obesidade e do sobrepeso na população adulta. É na fase adul- 
ta que doenças relacionadas ao sobrepeso e à obesidade matam milhões de pessoas todos os anos e afligem outros milhões, fazendo necessário o dispêndio de grande parte de recursos humanos, físicos e financeiros para o seu combate (GALATTI; PAES, 2008).

Para que se tenha o esporte como um hábito que se inicia na infância, é necessário que este seja apresentado como algo possível para a criança, com o qual suas conquistas sejam valorizadas, e não sua falta de habilidade seja um impedimento. E para que a prática esportiva contribua desde a infância para o controle de doenças decorrentes da obesidade, é importante o tratamento pedagógico adequado do esporte em um dos cenários possíveis para sua prática em larga escala: a escola. Como espaço de acesso democrático, aulas de Educação Física bem organizadas, sistematizadas, aplicadas e avaliadas podem apresentar o esporte de forma que os benefícios físicos, cognitivos, motores e afetivos sejam contemplados, em coerência com o projeto pedagógico. Nesse contexto, vários estudos (GOTMAKER et al., 1999; HUMBERT et al., 2006; GALATTI; PAES, 2008; Weintraub et al., 2008) destacam os JECs como modalidades importantes, por articular a prática esportiva com a convivência e o sentimento de pertencimento a um grupo.

Para tanto, Paes (2002), Galatti et al. (2008) e Galatti, Paes e Montero (2009) sinalizam para dois referenciais que devem ser contemplados nos programas de ensino e aprendizagem do esporte, inclusive no esporte escolar: um metodológico, ligado às questões técnico-táticas, e outro socioeducativo, na perspectiva da formação cidadã. Nesse conjunto os autores sinalizam para a pluralidade de possibilidades educacionais do esporte, tanto na sua prática através de diferentes modalidades e suas especificidades quanto na promoção e discussão de valores. Dessa forma, a prática pedagógica com o esporte está pautada na contribuição para a formação integral do indivíduo cujas necessidades devem ser contempladas, o que favorece a prática esportiva bem orientada como um hábito de vida saudável, em seu sentido amplo. Nessa perspectiva apresentaremos, na sequência, os JECs como um conjunto de modalidades especialmente favoráveis ao fomento da prática esportiva entre crianças e adolescente obesos e com sobrepeso. 


\section{Programas para a redução da obesidade infantil: perspectivas com base na prática dos JECs}

Considerando que a prevenção é a melhor situação para o combate da obesidade na infância, a literatura especializada demonstra preocupação com a proposição de programas bem-sucedidos. Em pesquisa de revisão bibliográfica em bases de dados internacionais, Mello et al. (2004) encontraram número significativo de trabalhos sobre obesidade infantil, com prevalência no Brasil. Entretanto, os autores constataram que poucos estudos trazem resultados de programas educativos aplicáveis no país, levando-os a concluir o seguinte: "Deve-se prevenir a obesidade infantil com medidas adequadas de prescrição de dieta na infância desde o nascimento, além de se estudar mais sobre programas de educação que possam ser aplicados no nível primário de saúde e nas escolas" (MELLO et al., 2004, p.173).

Para Weintraub et al. (2008), os programas para o tratamento clínico da obesidade infantil demandam grande quantidade de recursos, atendem a um número reduzido de crianças e não estão disponíveis em todas as comunidades, além de, geralmente, apresentarem resultados apenas modestos. Diante disso e do constante crescimento da obesidade infantil, os autores apontam para a necessidade de programas acessíveis e de melhor relação entre custo e benefício para ajudar crianças com sobrepeso no controle do problema.

Neste estudo, sinalizamos para o esporte - em especial os JECs como um elemento motivador e eficiente também no controle da obesidade, facilitando o atendimento a um maior número crianças. Weintraub et al. (2008) avaliaram a viabilidade, aceitabilidade e eficácia de um programa baseado na modalidade futebol no horário extraescolar, visando à redução de peso em crianças de baixa renda com sobrepeso. Após seis meses, o estudo constatou redução da massa corporal e incremento da atividade física diária, o que levou os autores a concluir que um programa extraescolar de prática regular do futebol pode ser acessível e eficiente como intervenção no controle do peso corporal na infância.

Com base nos resultados do estudo, Weintraub et al. (2008, p. 232) apresentam características da prática esportiva, sobretudo daquelas modalidades em equipe, que podem motivar crianças a praticar o exercício físico mais regularmente e com maior envolvimento afetivo: 
Playing sports; being part of a team; receiving mentoring, modeling, and friendship from young adult coaches; and having opportunities to demonstrate skills in front of friends and family may all be fun for children and thus highly motivating. ${ }^{1}$

Outro ponto levantado na literatura é a oferta de exercício físico para grupos específicos de crianças obesas, nos quais todas tenham semelhantes dificuldades e desafios relacionados ao sobrepeso. Em função disso, seria maior a possibilidade de haver nível semelhante de habilidades de jogo, o que facilitaria a demonstração de capacidades e habilidades, podendo as crianças obesas e com sobrepeso sentir-se mais à vontade entre outras crianças com o mesmo problema (HUMBERT et al., 2006; Weintraub et al., 2008).

Gotmaker et al. (1999) analisaram a implantação de um programa multidisciplinar para redução da obesidade em escolas de ensino fundamental nos Estados Unidos, o Planet Health. O programa durou 21 meses e buscou esclarecer o problema da obesidade entre os adolescentes, informando sobre hábitos saudáveis e estimulando sua adesão. Com a participação de 1.295 estudantes das sextas e oitavas séries de escolas públicas, o programa foi avaliado como bem-sucedido entre as meninas, enquanto não foram observadas baixas nos índices relacionados à obesidade entre os garotos. De forma geral, os pesquisadores sinalizam para o ambiente escolar como um espaço adequado para o combate à obesidade, já que todas as crianças americanas passam pela educação formal.

No Brasil a obesidade infantil também apresenta índices alarmantes, como vimos, e a escola se mostra o ambiente mais indicado para a instalação de políticas públicas que visem a combater o problema, uma vez que quase a totalidade das crianças brasileiras está na escola - como demonstra pesquisa divulgada em 28 de setembro de 2007 pelo IBGE, apontando que $97,6 \%$ das crianças e adolescentes entre sete e quatorze anos estavam na escola. É nessa faixa etária que se dão as primeiras experiências esportivas, sendo as competitivas indicadas para início entre dez e doze anos (ARENA; BÖHME, 2000).

1-Jogar esportes; ser parte de uma equipe; receber tutoria, modelação e aprendizagem de um jovem técnico adulto; e ter oportunidades de demonstrar habilidades diante de amigos e família pode ser divertido para crianças e ainda ser altamente motivador (tradução livre).

Pensar a Prática, Goiânia, v. 15, n. 2, p. 272-550, abr./jun. 2012 
Vale destacar que as crianças e adolescentes de zero a quatorze anos de idade representam $27,8 \%$ da população brasileira (IBGE, 2007). Quase metade $(48,9 \%)$ das famílias brasileiras, 28,9 milhões, tem crianças e adolescentes de até quatorze anos de idade. Em 2006, $40,4 \%$ dessas famílias foram consideradas pobres (com rendimento mensal per capita de até meio salário mínimo). Na faixa de rendimento mais alta (mais de cinco salários mínimos mensais per capita) havia, em 2006, apenas $2,2 \%$ de famílias com crianças até quatorze anos de idade em todo o território nacional. Dessa forma, um programa na escola democratizaria o acesso ao esporte e os benefícios dessa prática, quando bem orientada, para toda a população, independentemente da classe social. Nesse contexto, apontamos os JECs como um conjunto importante de modalidades, por facilitar o atendimento a grupos maiores, além de possibilitar intervenções pedagógicas também de caráter socioafetivo.

Considerando as diferentes razões pelas quais as crianças procuram a prática esportiva, o sucesso de programas para crianças com sobrepeso e obesidade nos Estados Unidos, sinalizado por Gotmaker et al. (1999) e Weintraub et al. (2008), e a gama cada vez mais ampla de atividades que crianças e adolescentes têm como opção de lazer na contemporaneidade, apontamos para a necessidade de compreender o esporte de forma ampla. É preciso expandir os significados de sua prática para além da formação de atletas, valorizando também a formação humana, para que desde a infância as pessoas se sintam atraídas e motivadas para a prática, podendo o esporte potencializar-se como um dos principais recursos de formação humana do atual século, sendo um agregado também no combate das doenças associadas à obesidade.

\section{Considerações finais}

O esporte é um fenômeno sociocultural contemporâneo de múltiplas manifestações, que agrega pessoas, divulga marcas, vende produtos, podendo ser relacionado tanto a grandes empreendimentos financeiros como a ações de cunho educativo. A obesidade tem também, no atual momento, alcance mundial, sendo considerada uma epidemia. Neste artigo, sinalizamos que esses dois fenômenos característicos deste século, o esporte e a obesidade, podem estar relacionados, sendo destacado o momento da infância. Se bem orientado e adequadamente oferecido desde a infância, com respeito à maturidade 
e às necessidades das diferentes fases de desenvolvimento biopsicossocial, o esporte - em especial os JECs - pode ser uma prática que acompanhará o indivíduo por toda a vida, podendo colaborar, inclusive, para o controle do sobrepeso e, por consequência, da obesidade. No entanto, processos de iniciação esportiva que desrespeitam a criança e a especializam precocemente através de atividades e cobranças similares às de atletas profissionais tendem a afastá-la dessa prática e de seus possíveis benefícios.

Assim sendo, defendemos processos de iniciação esportiva baseados em princípios pedagógicos que valorizem a infância, buscando em um primeiro momento ser prazerosos, para que o aluno se mantenha na prática esportiva, usufruindo os benefícios que esta pode acarretar para seu desenvolvimento integral. Defendemos, ainda, que se positiva, a prática esportiva tende a se manter como hábito por toda a vida, contribuindo para o controle de doenças ligadas ao sedentarismo, tal qual a obesidade, e outras relacionadas a esta, sendo um importante contributo para políticas de saúde coletiva.

Sport and obesity: relations for a health lifetime since children sport initiation

\begin{abstract}
Sport is one of the most important social phenomena of the contemporary world. Obesity and overweight are, in a similar way, treated also like a worldwide phenomenon, reaching epidemic levels. In this study, we point out the inadequate treatment of the sport during childhood, which may be a factor of distance between people and an active lifestyle. Furthermore, we pointed to the articulation of appropriate sports programs from infancy as a factor that may contribute to a lifestyle more healthy and active throughout life, being a controlling factor of the problem of obesity.
\end{abstract}

Keywords: Sport Pedagogy. Public Health. Obesity. Sport Initiation. Team Games.

Deporte y obesidad: perspectivas para un estilo de vida saludable a partir de la adecuada iniciación deportiva

\title{
Resumen
}

El deporte es uno de los fenómenos más extensos actualmente en todo el mundo. La obesidad y el sobrepeso, por su parte, también son un fenómeno mundial, alcanzando el status de epidemia. En este estudio mostramos que un tratamiento equivocado de la iniciación deportiva infantil puede mantener a los niños alejados de un estilo de vida más activo. Por otra parte, señalamos que la oferta adecuada de programas deportivos en la infancia puede resultar un factor favorecedor de un estilo 
de vida saludable a lo largo de toda la vida constituyendo, de este modo, un elemento de control de la obesidad y el sobrepeso.

Palabras clave: Pedagogía del Deporte. Salud Pública. Obesidad. Iniciación Deportiva. Deportes de Equipo.

\section{Referências}

ADAMS, M. H. et al. Obesity and blood pressure trends in rural adolescents over a decade. Pediatric Nursing Journal, v. 34, n. 5, p. 381-394, 2008.

ARENA, S. S.; BÖHME, M. T. S. Programas de iniciação e especialização esportiva na Grande São Paulo. Revista Paulista de Educação Física, São Paulo, v. 14, n. 2, p. 184-195, jul./dez. 2000.

$\mathrm{BAO}$ et al. Essential hypertension predicted by tracking of elevated blood pressure from childhood to adulthood: the Bogalusa Heart Study. American Journal of Hypertension, v. 8, p. 657-665, 1995.

BUNDRED, P. et al. Prevalence of overweight and obese children between 1989 and 1998: population based series of cross sectional studies. BMJ, v. 322, n. 7282, p. 313-314, 2001.

CINTRA, I. P. Avaliação da composição corporal e do gasto energético basal em crianças pré-púberes, com obesidade grave, na vigência de programa nutricional. 1999. $144 \mathrm{f}$. Tese (Doutorado em Medicina) - Escola Paulista de Medicina, Faculdade de Educação Física, São Paulo, 1999.

COAKLEY, J. Sport in society: issues and controversies. Dubuque: WCB/McGraw Hill, 1998.

DANIELS, S. R. Complications of obesity in children and adolescents. International Journal of Obesity, v. 33, supl. 1, p. 60-65, 2009.

DIETZ, W. H. Overweight in childhood and adolescence. New England Journal of Medicine, v. 350, n. 9, p. 855-857, 2004.

DURAZO-ARVIZU, R. A. et al. Rapid increases in obesity in Jamaica, compared to Nigeria and the United States. Bimedical Central Public Health, v. 8, n. 133, 2008. 
GALATTI, L. R. Esporte e clube sócio-esportivo: percurso, contextos e perspectivas a partir de estudo de caso em clube esportivo espanhol. 2010. 305 f. Tese (Doutorado em Educação Física) - Faculdade de Educação Física, Universidade Estadual de Campinas, Campinas, 2010. Disponível em: <http://cutter.unicamp.br/document/?co$\mathrm{de}=000770298>$. Acesso em: 10 jun. 2010.

GALATTI, L. R.; PAES, R. R. Sport and obesity: relations for a health lifetime since children sport initiation. Archivos de Medicina del Deporte, Pamplona, v. 25, p. 556-556, 2008.

GALATTI, L. R.; PAES, R. R.; MONTERO, S. A. Pedagogía del deporte y juegos deportivos colectivos: el juego como elemento en los programas a largo plazo de escuelas deportivas. Revista de Educación Física: Renovar la Teoría y Practica, A Coruña, v. 113, p. 13-18, 2009.

GALATTI, L. R. et al. Pedagogia do esporte: procedimentos pedagógicos aplicados aos jogos esportivos coletivos. Revista Conexões, Campinas, v. 6, n. especial, p. 404-415, 2008. Disponível em: $<$ http://polaris.bc.unicamp.br/seer/fef/viewarticle.php?id=319\&layout=abstract $>$. Acesso em: 15 jun. 2010 .

GORAN, M. I. Metabolic precursors and effects of obesity in children: a decade of progress, 1990-1999. American Journal of Clinical Nutricion, v. 73, p. 158-171, 2001.

Gortmaker, S. L. et al. Reducing obesity via a school-based interdisciplinary intervention among youth. Archieves of Pediatric and Adolescent Medicine, v. 154, p. 409-418, 1999.

HAMMER L. D. Obesidade. In: GREEN, M.; HAGGERTY, R. J. (Ed.). Pediatria ambulatorial. Porto Alegre: Artes Médicas, 1992. p. 440-455.

HEDLEY, A. A. et al. Overweight and obesity among US children, adolescents, and adults, 1999-2002. Journal of American Medical Association, v. 291, p. 847-850, 2004.

HUMBERT, M. L. et al. Factors that influence physical activity participation among high- and low-SES youth. Quality Health Research, v. 16, p. 467-483, 2006. 
IBGE. Pesquisa de Orçamentos Familiares 2002-2003: antropometria e análise do estado nutricional de crianças e adolescentes no Brasil. 2003. Disponível em: $<$ http://www.ibge.gov.br/home/estatistica/populacao/condicaodevida/pof/2003medidas/default.shtm>. Acesso em: 11 fev. 2010.

IBGE. Síntese de Indicadores Sociais: uma análise das condições de vida da população brasileira. 2007. Disponível em: $<$ http://www.ibge.gov.br/home/presidencia/noticias/noticia_visualiza.php?id_noticia=987\&id_pagina=1>. Acesso em: 1 fev. 2008 .

KAIN, J. et al. Obesity trends and determinant factors in Latin America. Caderno Saúde Pública, Rio de Janeiro, v. 19, supl. 1, p. 77-86, 2003.

LEE, Y. S. Consequences of childhood obesity. Annals Academy of Medicine Singapore, v. 38, p. 75-81, 2009.

LEONARD, H. E. et al. Decreasing sedentary behaviors in treating pediatric obesity. Archives of Pediatric and Adolescents Medicine, v. 154, p. 220-226, 2000.

MCARDLE, W. D. et al. Fisiologia do exercício: energia, nutrição e desempenho humano. 5. ed. Rio de Janeiro: Guanabara Koogan, 2001.

MELLO, E. D. et al. Obesidade infantil: como podemos ser eficazes? Jornal de Pediatria, Rio de Janeiro, v. 80, n. 3, p. 173-182, maio/jun. 2004.

MONTEIRO, C. A. et al. The nutrition transition in Brazil. European Journal of Clinical Nutricion, v. 49, p. 105-113, 1995.

NAGAI, N. et al. Trends in obesity among preschool and school children in administrative district of Hyogo Prefecture (Japan) 1981-2000. Japan Journal Nutrition Diet, v. 61, p. 189-194, 2003.

OGDEN, C. L. et al. Prevalence of overweight and obesity in the United States, 1999-2004. Journal of American Medical Association, v. 295, n. 13, p. 1549-1555, 2006. 
OLIVEIRA, C. L.; FISBERG, M. Obesidade na infância e adolescência: uma verdadeira epidemia. Arquivo Brasileiro de Endocrinologia Metabólica, v. 47, n. 2, p. 107-108, abr. 2003.

PAES, R. R. Aprendizagem e competição precoce: o caso do basquetebol. 3. ed. Campinas: Editora da Unicamp, 1997.

PAES, R. R. A pedagogia do esporte e os jogos coletivos. In: ROSE, J. R. Esporte e atividade física na infância e adolescência. São Paulo: Artmed, 2002. p. 89-98.

SANMARTÍN, G. M. El valor del deporte en la educación integral del ser humano. Revista de Educación, n. 335, p. 105-126, 2004.

SILVA, Y. P. G. et al. Pedagogia do esporte e métodos de ensino: entre a especialização e a iniciação precoce. In: CONGRESO INTERNACIONAL DE DEPORTES DE EQUIPO, 2., 2009, A Coruña. Anais... A Coruña: 2009.

STAMATAKIS, E. et al. Overweight and obesity trends from 1974 to 2003 in: English children: what is the role of socioeconomic factors? Archives of Disease in Childhood, v. 90, n. 10, p. 999-1004, 2005.

VELÁSQUEZ-MELÉNDES, G. et al. Epidemiologia do sobrepeso e da obesidade e seus determinantes em Belo Horizonte (MG), Brasil: uma transversal populacional estudo de base. Revista Panamericana de Salud Pública, v. 16, n. 5, p. 308-314, 2004.

WANG, Y.; MONTEIRO, C. A.; POPKIN, B. M. Trends of obesity and underweight in older children and adolescents in the United States, Brazil, China, and Russia. American Journal of Clinical Nutrition, v. 75, p. 971-977, 2002.

WEINTRAUB, d. L. et al. Team sports for overweight children: the Stanford sports to prevent obesity randomized trial (Sport). Archives Pediatrics Adolescents Medicine, v. 162, n. 3, p. 232-237, 2008.

WHITAKER, R. C. et al. Previsão da obesidade na idade adulta desde a infância e obesidade dos pais. English Journal Medicine, v. 337, n. 13, p. 926-927, 1997. 
WHO. World Health Organization. Disponível em: $<$ http://www.who.int/en/>. Acesso em: 9 jan. 2011.

Recebido em: 07/10/2010

Revisado em: 20/01/2011

Aprovado em: 07/06/2011

Endereço para correspondência

lagalatti@yahoo.com.br

Larissa Rafaela Galatti

Universidade Estadual de Campinas

Departamento de Ciências do Esporte.

Rua Érico Verrísimo 701

Barão Geraldo

13083-851 - Campinas, SP - Brasil

Caixa-Postal: 6134 